\title{
Supervivencia de trece años en una paciente con metástasis cutáneas aisladas de adenocarcinoma gástrico. ¿Ante qué enfermedad nos encontramos?
}

\author{
A. SEGURA HUERTA, J. A. PÉREZ-FIDALGO, P. LÓPEZ-TENDERO, R. GIRONÉS \\ SARRIÓ, J. APARICIO URTASUN
}

Servicio de Oncología Médica. Hospital Universitario La Fe. Valencia

\begin{abstract}
THIRTEEN YEARS SURVIVAL IN A PATIENT WITH ISOLATED SKIN METASTASES OF A GASTRIC CARCINOMA. WHAT KIND OF DISEASE IS THAT?
\end{abstract}

\section{RESUMEN}

El adenocarcinoma gástrico es un tumor con elevado porcentaje de recaídas y una alta letalidad. Peritoneo e hígado son las localizaciones más frecuentes de las metástasis.

Presentamos el caso de una paciente diagnosticada de adenocarcinoma gástrico que presentó una recaída aislada en piel con sucesivas recaídas a nivel cutáneo tratadas de forma local y con diversos esquemas de citostáticos. La paciente falleció 13 años después del diagnóstico de las metástasis.

La evolución de esta paciente sugiere la existencia de factores pronósticos biológicos en el carcinoma gástrico que influyen en el comportamiento de la enfermedad. El conocimiento de la biología tumoral puede aclarar los comportamientos atípicos de enfermedades que con los conocimientos actuales son similares pero cuya evolución es muy diferente.

PALABRAS CLAVE: Carcinoma gástrico. Metástasis cutáneas. Factores pronóstico. Largos supervivientes.

\begin{abstract}
The gastric adenocarcinoma is a high lethality tumour and has a great tendency to the recurrence. Liver and peritonea are the places where the metastases are most frequently localised.

We introduce the case of a woman diagnosed of gastric adenocarcinoma who showed isolated skin metastasis. There were an important number of recurrences (always in the skin). She was treated with radical surgery and later treated with different citostatic schedules. The patient died 13 years after metastasis were diagnosed.

With this case we wanted to pay attention about the role of the biologic prognostic factors of the gastric carcinoma. The molecular biology of these tumours can to explain the different evolution of the disease. Biologic prognostic factors can separate the gastric carcinoma into different kind of disease.
\end{abstract}

KEY WORDS: Gastric carcinoma. Skin metastases. Prognostic factors. Long time survival.

Segura Huerta A, Pérez-Fidalgo JA, López-Tendero P, Gironés Sarrió R, Aparicio Urtasun J. Supervivencia de trece años en una paciente con metástasis cutáneas aisladas de adenocarcinoma gástrico. ¿Ante qué enfermedad nos encontramos? An Med Interna (Madrid) 2003; 20: 251-253.

\section{INTRODUCCIÓN}

El cáncer gástrico es un tumor con alta letalidad. Dos tercios de los pacientes son irresecables en el momento del diagnóstico y el $80 \%$ de los que son resecados presentan posteriores recaídas (1). La supervivencia mediana de los enfermos metastáticos es de 10 meses y el porcentaje de supervivientes en esta situación oscila entre el 5 y el $15 \%$ a 5 años (2).

Presentamos el caso de una mujer con un carcinoma gástrico con estadio avanzado al diagnóstico y que tras ser gastrectomizada presentó sucesivas recidivas cutáneas que fueron tratadas repetidamente de forma radical. Nos encontramos ante una evolución atípica de esta enfermedad, tanto en la localización de la recaída como en el comportamiento poco agresivo del tumor reflejado en un tiempo de supervivencia muy prolongado.

\section{CASO APORTADO}

Una mujer de 61 años sin antecedentes de interés comienza con clínica digestiva consistente en dispepsia, meteorismo y molestias epigástricas ocasionales con irradiación en cinturón hacia región lumbar, no refiere pérdida de peso ni síndrome constitucional. Se le practica una endoscopia donde se observa un proceso ulceroso en incisura angularis a 3 centímetros del píloro que es biopsiado. El informe de la biopsia indica adenocarcinoma gástrico de células en anillo de sello. El estudio de extensión descartó la presencia de metástasis a distancia. En julio de 1984 es intervenida, practicándose

Trabajo aceptado: 16 de abril de 2002

Correspondencia: Ángel Segura Huerta. Servicio de Oncología Médica. Hospital Universitario La Fe. Avda. Campanar, 21. 46009 Valencia. e-mail: segura_ang @gva.es 
una gastrectomía parcial con reconstrucción tipo Billroth I. Se confirmó el diagnóstico histológico de adenocarcinoma infiltrante en todo el espesor de la pared y afectando la grasa subyacente, respetando bordes de resección, sólo un ganglio aislado negativo para tumor. Se consideró entonces un pT4pNxM0, estadio IIIA. No recibió tratamiento adyuvante.

En octubre de 1987 presenta una lesión cutánea que se extirpa y cuya anatomía patológica se informó como metástasis de adenocarcinoma de células en anillo de sello, tras la cirugía recibió radioterapia externa con electrones (64 Gy).

En los 8 años posteriores presentó otras cinco recaídas cutáneas todas ellas localizadas en el tercio superior de la espalda. Fueron extirpadas y tratadas con fluoropirimidas orales. En octubre de 1997 ante nueva progresión de la lesión cutánea se modificó el tratamiento citostático iniciándose etopósido oral. Un año después presentó gran progresión con afectación de toda la espalda. En ese momento se inició tratamiento con esquema FLEP (fluorouracilo, ácido folínico, etopósido y cisplatino) del que recibió 4 ciclos junto a cobaltoterapia (dosis de $30 \mathrm{~Gy}$ ). A principios de 1999 nueva progresión de las lesiones que prácticamente ocupan la mitad superior de la espalda y se extienden a cara anterior del cuello (Figs. 1 y 2). La paciente fallece en septiembre de ese mismo año a la edad de 76 años, 13 años después del diagnóstico de las metástasis. En ningún momento de la evolución presentó metástasis en otra localización.

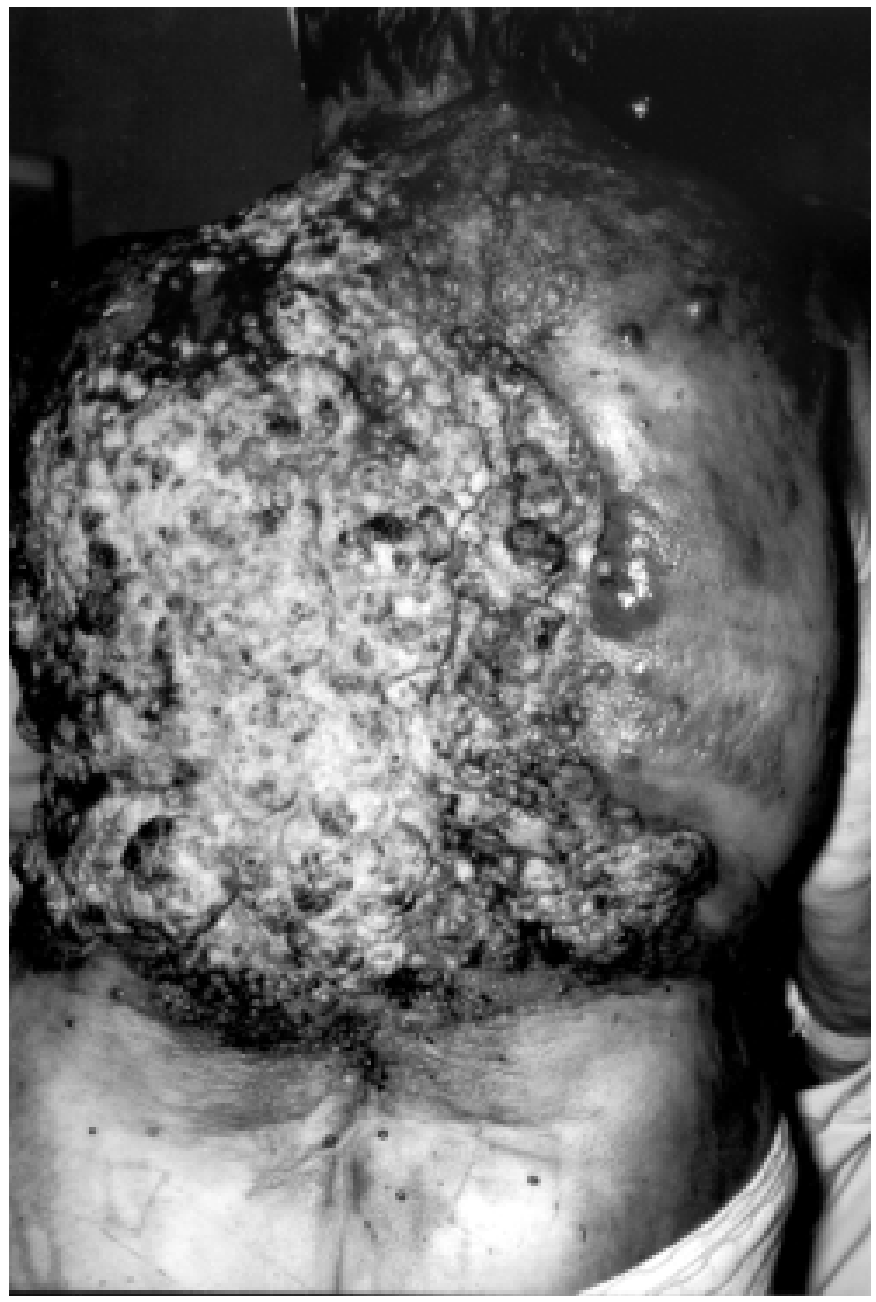

Fig. 1 .

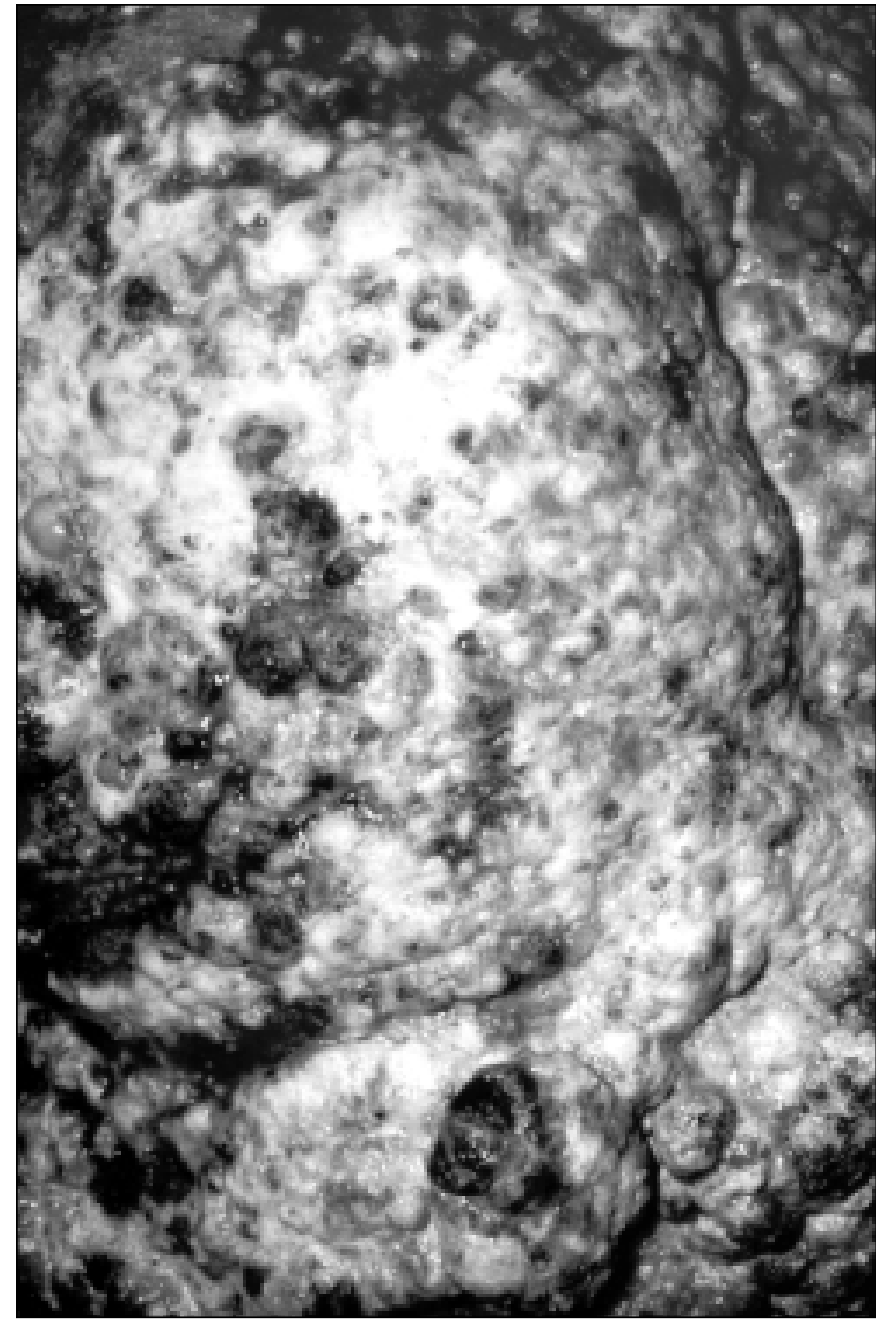

Fig. 2.

\section{DISCUSIÓN}

Las metástasis cutáneas son poco frecuentes en los carcinomas viscerales en general, especialmente en fases iniciales de la enfermedad. Suelen aparecer en fases finales de la enfermedad y se consideran de un pronóstico infausto (3-5). El cáncer gástrico es una enfermedad con una alta mortalidad, con supervivencias entre el 20-30\% a los cinco años (6). El procedimiento terapéutico de elección es la cirugía que sólo es posible de entrada en un tercio de los pacientes. La diseminación linfática es la que con más frecuencia se produce estando presente hasta en un $70 \%$ de los casos. La extensión por vía hematógena es sobretodo a hígado y menos frecuentemente a pulmón y glándula suprarrenal. Las metástasis a piel y partes blandas son un hecho poco frecuente (7).

En el momento actual los criterios de selección de grupos de riesgo se basan únicamente en el estadiaje. Los factores pronóstico más consolidados en el cáncer gástrico operado son la profundidad de la invasión de la pared y el número de ganglios afectos. También se conoce que los tumores de localización proximal tienen un pronóstico peor que los distales (8).

El comportamiento del proceso maligno de esta paciente nos hace pensar en la existencia de una serie de factores bioló- 
gicos propios del tumor que le hacen comportarse de una forma que no habitual. Han sido descritos varios factores pronóstico biológicos. La aneuploidía se asocia con mayor frecuencia a enfermedad locorregional avanzada (9). Otras anomalías genéticas asociadas a un peor pronóstico son la expresión de CD44, la reactivación de la telomerasa o la inactivación del gen p53 (10). Entre los factores pronósticos mejor estudiados se encuentran los inhibidores de las proteasas y las proteasas asociadas a tumores, las cuáles tienen un papel en el grado de invasividad del carcinoma gástrico (11). El sistema activador del plasminógeno se ha implicado en la capacidad invasora del tumor y las metaloproteinasas se implican en el desarrollo de metástasis a distancia (12).

La hipótesis de unas características biológicas específicas de este tumor explicarían tanto la aparición una metástasis aisladas en una localización atípica así como que en los siguientes años el carcinoma siguió recayendo únicamente en piel. La larga supervivencia de esta paciente viene matizada por la afectación únicamente de un órgano no vital. La escasa agresividad del tumor hace que deba considerarse una enfermedad muy distinta a la mayoría de adenocarcinomas gástricos metastáticos.

Este caso pone de manifiesto que determinados factores pronóstico biológicos o de factores predictivos de la respuesta al tratamiento pueden en un futuro ayudar a establecer el pronóstico e incluso seleccionar las secuencias terapéuticas en estos pacientes.

\section{Bibliografía}

1. Factores pronósticos del cáncer gástrico. En: González-Barón M. y cols (ed). Cáncer Gástrico. Documentos Consenso Oncología. Noviembre, 1999.

2. Roder JD, Bottcher K, Siewert JR y cols. Prognostic factors in gastric carcinoma. Results of the German Gastric Carcinoma Study 1992. Cancer 1993; 72: 2089-2097.

3. Muino Minguez A, Polo Casado A, Donis Sevillano E et al. Metástasis cutáneas como presentación de cáncer de origen desconocido. An Med Interna (Madrid) 1986; 10: 442-443.

4. Jurado Gámez B, Hidalgo Nuchera E, Merino Romero J. Metástasis cutáneas, presentación inusual del cáncer de pulmón. An Med Interna (Madrid) 1995;12: 346-348.

5. Franco Vicario R, Tirapu JM, Escalente J et al. Carcinoma epidermoide pulmonar en paciente de 22 años con metástasis cutáneas múltiples. An Med Interna (Madrid) 1998; 15: 591-593.

6. Mac Donald JS, Steele G, Gunderson L. Cancer of the stomach. En: DeVita VT, Hellman S, Rosemberg SA. Principles of Clinical Oncology.

7. Pestalozi BC, Von Hochstetter AR. Muscle metastasis as initial manifestation af adenocarcinoma of the stomach. Schweiz Med Wonchenschr 1998-00; 128: 38, 1414-7.

8. Kajiyama $\mathrm{Y}$, Tsurumaru M, Udagawa $\mathrm{H}$ et al. Prognostic factors in ade-
Otro punto de interés a discutir sería el tratamiento empleado en esta paciente. Dada la escasa frecuencia de aparición de metástasis cutáneas en el adenocarcinoma gástrico no está descrito en la bibliografía cuál es el tratamiento óptimo. Ciertos autores sugieren que la cirugía de las metástasis peritoneales así como de la enfermedad metastática hepática forma parte de los tratamientos que logran un mejor control de la enfermedad $(13,14)$. Se consideró en este caso que el tratamiento óptimo para las lesiones cutáneas sería fundamentalmente quirúrgico. No está claro ni siquiera para las metástasis hepáticas o peritoneales el papel de la quimioterapia tanto neoadyuvante como adyuvante (15). Sin embargo en enfermos con buen estado general puede valorarse el uso de quimioterapia oral con agentes como las fluoropirimidinas, el etopósido o incluso con esquemas más agresivos como el FLEP (fluorouracilo, ácido folínico, etopósido y cisplatino). Del mismo modo se podría justificar el empleo de radioterapia como un intento de asegurar el control de lesiones metastáticas locales y en zonas accesibles.

Nuestra paciente recibió tratamiento activo durante la mayor parte de los años de su evolución. El papel de dichos tratamientos en el control de esta paciente creemos que es indudable, a pesar de ello y dado el porcentaje de respuestas del adenocarcinoma gástrico a los citostáticos (alrededor del $20 \%$ ) consideremos que durante todos esos años estuvimos manejando una enfermedad conocida pero con unas características intrínsecas que desconocemos. nocarcinoma of the gastric cardia: pathologic stage anlysus and multivariate regression analysis. J Clin Oncol 1997; 15: 2015-2021.

9. Kimura H, Yonemura Y. Flow cytometric analysis of nuclear DNA content in advanced gastric cancer and its relation to prognosis. Cancer 1991; 67: 2588-2593.

10. Tahara E, Semba S, Tahara H. Molecular Biological observations in gastric cancer. Semin Oncol 1996; 23: 307- 315.

11. Heiss MM, Babic R, Allgayer H y cols. Tumor-associated proteolysis and prognosis: new functional risk factors in gastric cancer defined by the urodinase-type plasminogen activator system. J Clin Oncol 1995; 13: 2084-2093.

12. Nekarda H, Schmitt M, Ulm K y cols. Prognostic impact of urokinasetype plasminogen activator and its inhibitor PAI-1 in completely resected gastric carcinoma. Cancer Res 1994; 54: 2900-2907.

13. Yonemura Y, Fujimura T, Fushida S y cols. Peritonectomy for advanced gastric cancer. En : Yutaka Y (ed.): Peritoneal dissemination. Kanazawa, Japón: Maeda Shoten Co Ltd 1998; 275-293.

14. Ochiai T, Sasako M, Mizumo S y cols. Hepatic resection for metastatic tumours from gastric cancer: analysis of prognostic factors. Br J Surg 1994; 81: 1175-1178.

15. Lindell G, Ohlsson B, Saarela A y cols. Liver resection of non-colorectal secondaries. J Surg Oncol 1008; 69: 66-70. 